Original scientific article

UDC: 613.94(091)(485)

COBISS.SR-ID 219480588

\author{
Alessandro Berlini \\ University of Naples "L'Orientale" \\ Via Chiatamone 61/62, 80121 Napoli, Italy
}

\title{
EUGENICS AND STERILIZATION POLICIES: THE CASE OF TATTARE
}

\begin{abstract}
In the first decades of twentieth century, eugenic theories were used by some of the Western countries to justify actions of biopolitics toward various categories of persons defined as "degenerated" (feeble-minded, criminals, ethnic minorities, "morally irresponsible" persons, alcohol and drugs addicted, vagrants, etc). One of the cruelest actions was the compulsory sterilization policies toward these categories, to whom was denied the right to have child in the name of "racial integrity" of the social body. Not only in Nazi Germany, but also, in the United States, Canada, Switzerland, and in the countries from Scandinavian area, Roma and other groups of nomads by similar customs were victims of these policies. Since second half of the nineteenth century, scholars in eugenics and criminology defined them as "hereditary wanderers" or "born criminals". The article will focus on the case of Tattare, a group of nomads settled in Sweden and Norway since the sixtieth century, which was targeted by Swedish eugenic law. Between 1920 and 1950, Swedish scientists tried to prove the "racial inferiority" of Tattare, while politicians and economists stressed the impossibility of their integration in the Swedish society, and their condition of parasitism. Therefore, between 1935 and 1960, Swedish law included the Tattare in the eugenic national program of compulsory sterilization, contributing to their ethnocide.
\end{abstract}

Keywords: eugenics, sterilization, Sweden, Roma

Non MeSH: Tattare, antiziganism

\section{Teorie e pratiche dell'antiziganismo}

Leugenetica nasce in parte rifacendosi alle teorie elaborate, nella seconda metà del XIX secolo, da intellettuali italiani appartenenti alla scuola della Sociologia e dell'Antropologia criminale. Tra questi, la figura di maggior rilevanza fu Cesare 
Lombroso. La sua opera principale, L'uomo delinquente, e la sua teoria della degenerazione umana, furono per decenni punto di riferimento a livello internazionale per antropologi, psichiatri, alienisti, criminologi, e teorici dell'eugenetica.

Per Lombroso il delinquente-nato è una regressione degenerativa ad uno stadio antecedente la civiltà, selvaggio quando non animalesco; il criminale è la scimmia in mezzo a noi, e Lombroso lo paragona al parassita che succhia la linfa della pianta sulla quale vegeta, perché interviene a distruggere i frutti del progresso della società. Le anomalie identificate nel criminale sono, a parere di Lombroso, le stesse che è facile ritrovare nel selvaggio, e questo ritorno ad una "animalità anteriore" si misura essenzialmente nel fatto che in entrambi mancano la pietà, lonestà, il senso del giusto e del delitto. Unica logica e possibile soluzione auspicata per estirpare definitivamente dalla società il morbo criminale era ritenuta la soppressione fisica del delinquente-nato.

Le stesse caratteristiche degenerative attribuite al delinquente nato furono attribuite, da Lombroso e dai suoi epigoni contemporanei, agli Zingari. Essi furono definiti come "un'intera razza di delinquenti", che riproduceva a livello di organismo collettivo tutti i vizi e le passioni che era possibile individuare nel "delinquente nato". "Gli Zingari hanno l'imprevidenza sia del selvaggio che del criminale. [.........] Divorano carogne mezze putrefatte. Sono dediti a orge, amano il rumore e fanno un grande clamore nei mercati. Uccidono a sangue freddo per rubare e sono sospettati di cannibalismo" [1; III].

Anche Giuseppe Sergi contribuì al fermento ideologico in questione. Nel suo Le degenerazioni umane (1889), dissertando a proposito di mendicanti e vagabondi, definì gli Zingari una "razza selvaggia", e, come gli altri vagabondi, testimonianza di un esempio involutivo della civiltà, della quale non condividerebbero il costume del lavoro e della sedentarietà, essendosi in essi fissata atavicamente la tendenza a vagare, all'inerzia fisica, spirituale ed intellettuale, alla criminalità:

Che dal vagabondaggio e dalla mendicità si vada alla delinquenza, è intelligibile, perché colà ove non esiste carattere e condotta normale, la delinquenza ha la sua origine naturale. Così che, per noi, vagabondi e mendicanti di qualunque tipo costituiscono una classe di esseri degenerati, che manifesta la sua condizione d'inferiorità nella lotta per l'esistenza nell'ambiente sociale $[2$, p. 176].

In Italia la costruzione di questo stereotipo trovò il suo culmine ne Gli Zingari. Il problema di una gente vagabonda in lotta con le leggi, del giudice napoletano Alfredo Capobianco (1914).

La lascivia, gli zigomi sporgenti, l'idioma selvaggio, l'avversione al lavoro, la delinquenza catalizzata da un istinto innato, i tatuaggi, il naso aquilino, la sporcizia, il cannibalismo, l'insensibilità al dolore o al freddo, la barbarie morale, l'inconsistenza di una tradizione intellettuale, la tendenza alla prostituzione delle donne: questi sono solo alcuni del profluvio di pregiudizi riversato da Capobianco sugli Zingari nella prima parte del libro. 
Secondo Capobianco, oltre ad essere dettata da motivi di ordine interno, vista la pericolosità sociale degli Zingari, l'avversione a questa "razza" sarebbe giustificata anche dal punto di vista morale, essendo generata dall'enorme distanza che separa la civiltà dalla bestialità degli Zingari. Ciò legittimerebbe provvedimenti legislativi repressivi e restrittivi, come vere e proprie leggi speciali atte ad impedire l'immigrazione degli Zingari, o l'inasprimento ad hoc delle pene per i reati da essi commessi. Tra di esse, la condanna per i delinquenti zingari alle colonie penali ed ai lavori forzati, una pena "tremenda per gente abituata a vagare ed a non lavorare":

Di fronte allattività criminosa e diabolica degli Zingari, occorrono norme speciali, mezzi adeguati. [........] E soprattutto occorre l'opera del legislatore. $[\ldots \ldots \ldots .$.$] Or, la mala vita zingaresca in Italia è una piaga anche$ della nostra epoca. È una vera poltroneria sospetta, che passeggia su e giù in cerca di un buon colpo ed eliminarla è un utilissimo atto sociale. Ma non la si può eliminare, se non la si previene con apposite disposizioni e con buone norme [3, pp. 31-92].

Venendo più direttamente all'ambito delleugenetica, e delle teorie al suo interno elaborate a riguardo degli Zingari, dobbiamo fare innanzitutto riferimento a Charles B. Davenport, figura leader della comunità eugenetica degli Stati Uniti nei primi decenni del XX secolo.

The Feebly Inhibited, pubblicato nel 1915, è il testo che forse meglio di tutti riassume la teoria della degenerazione ereditaria di Davenport. Ma la lettura di uno dei capitoli di questo testo, quello dedicato al nomadismo, apre anche la strada ad una rivalutazione dell'originalità e del peso che ebbe l'eugenetica americana nel formulare ipotesi che più avanti sarebbero state riproposte in Europa con finalità pratiche esplicitamente razzistiche. Quello che dalla scienza nazista, grazie al lavoro di Robert Ritter e della sua assistente Eva Justin, fu battezzato come Wandertrieb [4, p. 352], la tendenza innata al nomadismo, ed utilizzato per legittimare "scientificamente" il genocidio degli Zingari, era in realtà già stato teorizzato da Davenport oltre vent'anni prima [5, pp. 9-27]. Egli aveva parlato di "Wandering instinct", istinto al nomadismo, responsabile del quale sarebbe stato un gene specifico.

Questa inclinazione istintiva a vagare era ritenuta universale caratteristica della specie umana, ma secondo Davenport essa, negli individui normali, era inibita e soffocata dallistinto alla socialità ed alla sedentarizzazione. Viceversa, una degenerazione ereditaria conduceva alcuni individui ad un patologico nomadismo perpetuo, che si tramandava di generazione in generazione, ed era assimilabile o ad un arresto di sviluppo o ad un primitivismo atavico, avendo Davenport riscontrato l'istinto al nomadismo sia nei bambini, sia nelle scimmie antropomorfe. Lassenza del "gene della domesticità" sarebbe stata più che mai evidente tra gli Zingari, definiti "nomadic race", la cui endogamia avrebbe contribuito a paralizzare l'istinto stanziale e a trasformare un intero popolo in una massa errante, sempre sfuggente al tentativo di qualsiasi legislatore di fare insediare in un luogo questi soggetti. Il nomadismo degli Zingari testimoniava del loro essere una "razza passiva che non riconosce nessuno dei vincoli imposti dai dieci comandamenti"; un insieme di epilettici e 
depravati, sporchi e dipsomani. Tramite lo studio di un centinaio di casi, Davenport mise così in relazione il nomadismo con tutta una serie di degenerazioni secondarie, che pur emergendo singolarmente o rimanendo allo stato latente, sarebbero tutte contenute in nuce nel patrimonio cromosomico; facile, allora, trovare nella stirpe nomade criminali, alcolizzati o "very good women".

A parte Davenport, anche altri eugenisti americani dellepoca insistettero nel mettere in luce la presunta relazione tra patologia, ereditarietà e nomadismo. Cresciuti all'ombra dell'Eugenic Record Office, fondato dallo stesso Davenport nel 1906, autori come Laughlin, Popenoe e Goddard sottolineavano la pericolosità per il patrimonio razziale americano di girovaghi e vagabondi (categorie in cui rientravano anche gli Zingari americani), e la necessità di misure draconiane atte ad ostacolare il loro stile di vita, quando non ad impedire loro la stessa riproduzione. In molti dei circa trenta degli Stati Uniti che, a partire 1907 dell'Indiana nel 1907, promulgarono leggi riguardanti la sterilizzazione eugenetica, ad essere oggetto della legge furono anche queste categorie di persone.

Sulla scia degli sviluppi delleugenetica statunitense, anche in Europa allinizio del Novecento furono creati una serie di Istituzioni scientifiche rivolte ad approfondire questioni legate alleugenetica, e ad elaborare misure atte a prevenire il moltiplicarsi di categorie di soggetti definiti come "degenerati". Tra loro, anche gli Zingari furono oggetto di ricerca, stigmatizzati come soggetti "irrecuperabili" e un pericolo per la qualità biologica e l'ordine sociale delle popolazioni europee. In Germania, ad esempio, fin dai prima anni del ' 900 alla schedatura poliziesca di Rom e Sinti presenti in territorio tedesco si affiancarono una serie di ricerche su questi gruppi, che ne dimostravano l'inferiorità razziale e le irreversibili predisposizioni ereditarie alla delinquenza e alla devianza.

In questo contesto, andò affermandosi la figura di Robert Ritter, che diventerà il principale ideologo della persecuzione nazista degli Zingari. Attivo già negli anni '20, Ritter aveva condotto, a partire dal 1932, una serie di ricerche su Zingari, criminali e vagabondi, al fine di studiarne il background familiare, e nel 1936 gli venne affidata la direzione dell' Istituto di ricerca per l'Igiene Razziale e l'Ereditarietà. La ricerca si protrasse per anni, data la vastità dellimpresa. Ritter si era difatti proposto di studiare e schedare tutti gli Zingari presenti sul suolo germanico, al fine di evidenziare dei caratteri distintivi che accomunassero tutti i soggetti osservati, e potere "scientificamente" dimostrare la devianza dell'intero gruppo.

Il lavoro di Ritter e della sua equipe culminò nell'individuazione del Wandertrieb, il gene responsabile di un innato "istinto al nomadismo" nella popolazione zingara, grazie soprattutto al lavoro della sua collaboratrice Eva Justin [4, pp. 352-354]. Stigmatizzandola contemporaneamente come una "sottorazza" corrotta da millenni di vagabondaggio e promiscuità e insieme come Asozialen fatalmente inclini alla delinquenza e alla trasgressione delle leggi, e raccomandandone la sterilizzazione integrale [6, p. 369]. Già dal 1930 il giurista Hans Frank aveva scritto che gli unici modi per liberarsi di questa ampia categoria di soggetti (che comprendeva i criminali, i mendicanti, i vagabondi, gli alcolizzati, le prostitute) erano l'esilio o "l'eliminazione della loro capacità riproduttiva". Ecco la "diagnosi” e la "prognosi" di Ritter: 
"Per incrocio razziale indigeno, gli Zigani si mescolano prevalentemente con vagabondi, asociali, criminali, ed a causa di ciò si è prodotto un sottoproletariato di Zigani e vagabondi. [ .] Come ulteriore risultato della ricerca, abbiamo osservato che gli Zigani sono del tutto primitivi dal punto di vista etnologico, ed il loro ritardo spirituale li rende incapaci all'adattamento sociale. [ ] La questione zigana potrà dunque considerarsi risolta, solo quando il grosso degli ibridi Zigani, asociali e fannulloni, sarà riunito in grandi campi ed ivi indotto al lavoro, e quando l'ulteriore aumento di questa popolazione mista sarà definitivamente impedito" [7, pp. 70-71].

E' incerto il numero di Zingari che furono effettivamente sterilizzati nel periodo nazista. Tra le categorie di individui "disgenici" cui era rivolta la legge nazista sulla sterilizzazione eugenetica, del 14 luglio 1933, non rientravano esplicitamente gli Zingari. Di fatto però essi vennero fatti rientrare in categorie cui la legge era rivolta, come "criminali ereditari", "frenastenici", o "vagabondi psicotici", il che li rendeva a pieno titolo candidati alle sterilizzazioni. Più tardi, la persecuzione le sostituirà con l'internamento degli Zingari, e la loro eliminazione fisica nei campi di concentramento. Qui gli Zingari, in particolare i bambini, costituiranno il gruppo "privilegiato" da sottoporre ad atroci esperimenti.

Va detto, peraltro, che prima ancora della legge eugenetica nazista, gli Zingari erano stati altrove sottoposti ad analoghi provvedimenti. Ad esempio in Svizzera, dove a livello cantonale esistevano alcune leggi sulla sterilizzazione eugenetica già dal 1928, ad essere colpiti furono gli Jenish. Conosciuti anche come "Zingari bianchi" (la loro origine infatti non è indiana, come nel caso di Rom e Sinti, ma germanica), si caratterizzavo per il loro nomadismo. Nel loro caso, le sterilizzazioni forzate si univano ad altre misure persecutorie pianificate, a partire dal 1926, per sradicare dal territorio elvetico il nomadismo. Queste vessazioni, più accentuate nel Ticino e nella Svizzera tedesca, e attuate fino al 1972, si realizzarono attraverso una serie di politiche preposte ad imporre una sedentarizzazione forzata, al fine di trasformare e distruggere col tempo le abitudini legate alla vita nomade, trasformando di fatto gli zingari in cittadini "normali": le sterilizzazioni, appunto, rientravano in questo programma di genocidio culturale. Ad esse si affiancò il divieto, per gli Jenish, di sposarsi tra loro, e la sistematica sottrazione dei bambini ai loro genitori naturali nell'ambito del progetto denominato "Kinder der Landstrasse" ("bambini della strada"), ideato e diretto da Alfred Siegfried. Bambini che venivano affidati ad istituti o a famiglie svizzere, di modo che acquisissero un comportamento consono al modello imposto, basato sui principi dell'ordine e del lavoro. Un'ulteriore forma di persecuzione fu l'internamento degli Jenish, in quanto "classe pericolosa", o in quanto psicopatici. In questo, l'Opera fu supportata dal lavoro compiuto dagli scienziati svizzeri negli anni '20 e '30; che, pienamente concordi col pregiudizio tradizionale della criminalità degli Zingari e con l'ideologia degenerazionista, finirono per abbracciare apertamente la teoria del nomadismo innato. "Il loro stile di vita, incompatibile con i principi morali della società borghese, che vedeva nella vita errabonda la via verso il crimine, doveva quindi essere normalizzato" [8, p. 3]. 


\section{Svezia, un'eugenetica "socialdemocratica”. Il caso dei Tattare}

Già dalla fine del XIX secolo gli scienziati svedesi parteciparono al dibattito europeo ed americano sull'ereditarietà e sulla degenerazione. Precursore di questi studi fu Hermann Lundborg, che a partire dal 1898 portò avanti studi su alcune famiglie della campagna svedese, evidenziando la presenza di gravi patologie che si trasmettevano nei loro membri generazione dopo generazione. A partire dai primissimi anni del Novecento sarà la città di Uppsala il fiore all'occhiello della scienza eugenetica svedese: presso la sua Università insegnavano, oltre a Lundborg, altri eminenti scienziati svedesi, i quali contribuiranno in maniera determinante alla fondazione, nel 1921, dell'Istituto svedese di biologia razziale, proprio a Uppsala [9; II, pp. 448-452].

La Svenska Sällskapet för Rashygien (Società svedese di igiene razziale) venne creata a Stoccolma nel 1909. Intanto, gli eugenisti svedesi costituivano già il principale gruppo straniero accreditato presso la società eugenetica internazionale con sede a Berlino [10]. Per iniziativa dei socialdemocratici, ma ampiamente sostenuti dai conservatori, e col supporto del nucleo scientifico di Uppsala, il Riksdag istituì poi l'Istituto svedese di biologia razziale, ideato da Alfred Petrén, e diretto a lungo proprio da Lundborg. Un organo unico al mondo nel suo genere fino a quel momento, e della cui organizzazione e struttura i tedeschi riprodurranno un modello su più vasta scala, creando nel 1927 il "Kaiser Wilhelm Institut für Antropologie, menschliche Erblehre und Eugenik". Già durante le discussioni parlamentari, che portarono all'atto di costituzione dell'istituto, si ventilava da parte socialdemocratica la necessità di indirizzarlo verso la legittimazione scientifica di una politica di sterilizzazione coatta che facesse degli svedesi una "razza di valore", attraverso "un po' di selezione, esattamente come facciamo con gli animali" [11, pp. 327-328].

Il lavoro dei collaboratori dell'istituto si concentrò, in primo luogo, su una schedatura antropometrica e genetica della popolazione svedese, al fine di identificare i ceppi razzialmente puri e quelli imbastarditi, di esaminare il rapporto esistente tra patologia ed ereditarietà, e di capire se e come esso sarebbe potuto degenerare nella contaminazione dei soggetti sani. Alla fine del 1923 vennero rese note le statistiche riguardanti l'osservazione di 76.000 soggetti, tra militari, studenti, ed internati in ospedali, manicomi, carceri. Da notare che l'istituto lavorò fin dalle origini, attraverso misurazioni craniologiche, e nel senso della stigmatizzazione degli Zingari, e in quello della "desvedesizzazione" delle minoranze lapponi e finlandesi che vivevano in territorio svedese, alle quali venivano associati tratti "mongoli", ritenuti similari a quelli di una razza inferiore [9; II, p. 433]. Non va tuttavia sopravvalutato il ruolo che ebbe il lavoro dell'istituto di Uppsala nel condizionare i successivi risvolti legislativi, che vanno viceversa addebitati soprattutto a scelte politiche [12, p. 358].

Una prima legge che prevedeva la sterilizzazione di soggetti definiti vagamente come "idioti" ("Sinneslöa”) fu promulgata nel 1934. Negli stessi anni, anche Danimarca, Finlandia e Norvegia si dotarono di normative analoghe. Una nuova 
legge, nel 1941, estese in Svezia le categorie di soggetti passibili di sterilizzazione. Essa, in particolare, si rivolgeva anche contro gli "asociali", partendo dal presupposto del rapporto deterministico tra "asocialità" e presenza di tare "razziali"ereditarie, fisiche o psichiche che fossero. Un escamotage utilizzato solo per preservare il substrato di legittimità scientifica che aveva inizialmente sostenuto la legislazione eugenetica. Anche quando sono gli Zingari ad essere presi di mira per essere colpiti dalla legge, la stigmatizzazione razziale è subordinata alla valutazione del loro modus vivendi, evidentemente poco conciliabile con la mentalità ritenuta "normale" nel modello svedese. Il pretesto "ufficiale" per il loro tentato etnocidio fu la loro presunta incapacità di potersi prendere cura di eventuali figli [10, pp. 124130]. Ecco il giudizio del deputato socialdemocratico Hedlund, esposto al Riksdag durante i dibattimenti del 1941, a proposito della necessità di estendere la norma sulla sterilizzazione svincolandosi da una concezione rigidamente biologistica del concetto di degenerazione:

"Io condivido il giudizio della commissione, riguardo al fatto che una condotta di vita asociale si trova spesso in una certa relazione con disturbi mentali. Penso in modo particolare agli alcolizzati, ai vagabondi, agli Zingari (Tattare) e ai delinquenti abituali: ma in queste categorie possono darsi casi di comportamento asociale senza che -almeno per i medici- sia possibile individuare segni di un disturbo mentale. Ci sono cioè dei casi, in cui la malattia mentale presso l'asociale ha un carattere secondario. Io non capisco perché queste persone debbano poter mettere al mondo dei figli. Nelle città più grandi conosciamo bene quel tipo di asociale, che generazione dopo generazione vive da parassita nella società. Persone di questo tipo hanno un sacro terrore per il lavoro, e cercano con ogni mezzo di farsi mantenere" [11, p. 342].

La Svezia aveva conosciuto, a partire dal 1914, un periodo di recrudescenza legislativa nei confronti degli Zingari. A quell'anno risale un bando totale all'ingresso di popolazioni nomadi nel paese, che verrà rimosso solo quarant'anni più tardi [13, p. 54]. Con queste premesse, gli Zingari vennero additati anchessi come asociali, e accusati di essere degli "improduttivi" per eccellenza, dato il loro rifiuto di lavorare nelle fabbriche o di abitare nei quartieri dormitorio. Contemporaneamente, se ne metteva in risalto la propensione etnica alle attività illecite. Un articolo pubblicato nel supplemento domenicale del Dagens Nyheter del 5 dicembre 1935, ad esempio, ironizzava sul fatto che, malgrado gli Zingari, per effetto dei loro ladrocini, disponessero ormai di "automobili di lusso e pellicce", l'abitudine atavica a condizioni di vita animalesche portava "marito, moglie e 11 figli" a vivere in una sola stanza......

Furono in molti a sollecitare unestensione delle sterilizzazioni anche ai Tattare. Con questo termine venivano etichettati i mercanti e castratori di cavalli appartenenti ad un gruppo seminomade presente nellarea scandinava fin dal XVI secolo. Il censimento nel 1942 ne stimò il numero in 7.668 [13, pp. 129-139]. I coniugi Myrdal, premi Nobel, li classificarono come "semi-idioti e palesemente 
inadatti come genitori". Va peraltro ricordato che i Tattare erano stati schedati come "razzialmente alieni" già dal gruppo dei collaboratori dell'istituto di Uppsala, e stigmatizzati da Lundborg come "vagabondi e criminali" [14, p. 216].

Anche se si dichiarava da parte socialdemocratica che ci si trovava di fronte ad una questione "prettamente razziale", nella realtà il nucleo del problema era costituito dalla marginalità dei Tattare. A testimonianza di ciò il fatto che, nonostante l'Istituto di biologia razziale di Uppsala, con uno studio del 1945, avesse sancito l'impossibilità scientifica di distinguere con certezza unorigine di stirpe Tattare da quella del resto della popolazione svedese, le sterilizzazioni dei membri di questo gruppo continueranno almeno fino agli anni Cinquanta, con motivazioni come questa, riferita al caso di una diciassettenne sterilizzata nel 1943:

"Scura, tipico aspetto Tattare....... Tipica mentalità Tattare: evasiva, bugiarda e codarda” [10, p. 129].

A seguito dell'approvazione della legge del 1941, il numero degli interventi crebbe a dismisura, essendosi evidentemente moltiplicate le fasce di popolazione che avrebbero potuto essere equiparate ai "Sinneslöa" (un'inchiesta del 1946 stima il numero dei potenziali candidati alla sterilizzazione in circa 340.000 individui). La moltiplicazione delle sterilizzazioni si spiega anche per effetto dell'annullamento di tutte le pur minime garanzie a favore del paziente che la legge del 1934 aveva preservato. Eliminata la possibilità di sfuggire all'intervento tramite un qualsiasi cavillo, il Riksdag, sostenuto unanimemente nell'iniziativa da maggioranza ed opposizione, decretò che il criterio della "permanenza" della malattia sarebbe dovuto scomparire, sostenendo viceversa che sarebbe bastata l'osservazione limitata nel tempo di un qualunque comportamento degenerato od asociale, da parte non solo di un medico, ma di qualsiasi cittadino (vicini di casa, insegnanti, parenti), per compromettere seriamente la posizione del soggetto e far avviare le pratiche per la sterilizzazione. Le autorità locali vennero così direttamente investite del potere di regolare e giudicare, nel contesto di loro competenza, la vita sessuale e familiare delle persone, e questa libertà si realizzava prima di tutto nel tentativo di ridurre le spese sociali del distretto riducendo gli assegni di maternità, oltre che nella velleità di stimolare una cultura della conformità alle regole. E, dopo il 1945, gli interventi furono rivolti quasi esclusivamente a donne, madri "troppo prolifiche" o ragazze "sessualmente pericolose", ritenute indegne del sistema svedese [12].

Quanto ai Tattare, continuarono ad essere stigmatizzati come un sottoprodotto della stirpe svedese; per continuare a sorreggere la politica eugenetica nei confronti della Tattarplågan, la burocrazia svedese continuò a sostenerne l'origine etnica aliena. Un uso massiccio dei test d'intelligenza venne fatto, durante tutto il periodo della persecuzione, per sanzionare l'irresponsabilità dei Tattare, e quindi la necessità della loro sterilizzazione. Di fatto disgregati come gruppo separato dall'ostilità nei loro confronti, la soluzione al problema si è realizzata nella "invisibilità sociale" dei Tattare. 


\section{Bibliografia}

1. Lombroso C. L'uomo delinquente in rapporto alla Antropologia, alla giurisprudenza ed alla Psichiatria. V ediz., 3 vol. Torino: Bocca; 1896.

2. Sergi G. Le degenerazioni umane. Milano: Dumolard; 1889.

3. Capobianco A. Gli Zingari. Il problema di una gente vagabonda in lotta con le leggi. Napoli: Raimondi; 1914.

4. Friedlander H. Le origini del genocidio nazista. Roma: Editori Riuniti; 1997.

5. Davenport CB. The feebly Inhibited. Washington: Carnegie Institute; 1915.

6. Tyrnauer G. Mastering the Past. Germans and Gypsies. U: Chalk F, Jonassohn K, urednici. The History and Sociology of Genocide. Analyse and Case Studies. New HavenLondon: Yale University Press; 1990. 366-377.

7. Muller-Hill B. Scienza di morte. L'eliminazione degli Ebrei, degli Zigani e dei malati di mente 1933-1945. Pisa: ETS; 1989.

8. Jourdan L. Caccia agli Zingari in Svizzera. Eugenetica in Europa tra le due guerre ed oltre. Le Monde diplomatique (ediz. italiana a cura de Il Manifesto). 1999 Ottobre; p. 3.

9. Nisot MT. La question eugénique dans les divers pays. 2 vol. Bruxelles: Falk Fils; 1927 1929.

10. Broberg G, Tydén M. Eugenics in Sweden: Efficient Care. U: Broberg G, Roll-Hansen N, urednici. Eugenics and the Welfare State. Sterilization Policy in Denmark, Sweden, Norway and Finland. East Lansing: Michigan State University Press; 1996. pp. 77-149.

11. Colla PS. La politica di sterilizzazione in Svezia 1934-1975. Rivista di storia contemporanea 1996;3:323-348.

12. Runcis M. Steriliseringar i folkhemmet. Stockholm: Ordfront; 1998.

13. Colla PS. Per la nazione e per la razza. Cittadini ed esclusi nel "modello svedese". Roma: Carocci; 2000.

14. Lundborg H, Runnström J. The Swedish Nation in Word and Picture. Together with Short Summaries of the Contributions Mades by Swedes within the Fields of Anthropology, Race-Biology, Genetics and Eugenics. Stockholm: Tullberg; 1921.

Submitted : 3. 11. 2015.

Reviewed: 13. 11. 2015.

Accepted : 19. 11. 2015. 